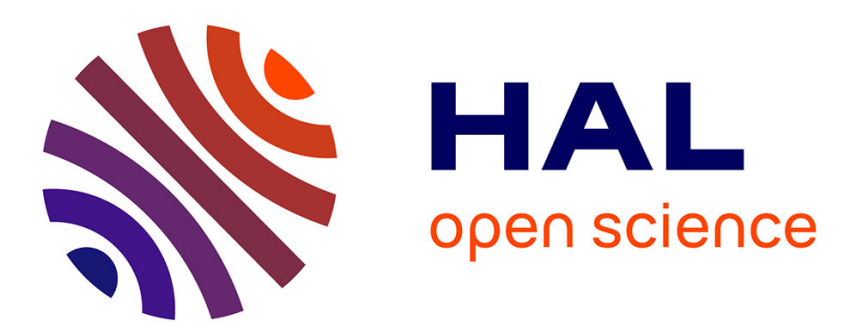

\title{
Characterizing the Nature of the Smectic A-Smectic C and Smectic A-Smectic C* Transitions
}

Ludovic Benguigui, P. Martinoty

\section{To cite this version:}

Ludovic Benguigui, P. Martinoty. Characterizing the Nature of the Smectic A-Smectic C and Smectic A-Smectic C* Transitions. Journal de Physique II, 1997, 7 (2), pp.225-228. 10.1051/jp2:1997120 . jpa-00248438

\section{HAL Id: jpa-00248438 https://hal.science/jpa-00248438}

Submitted on 1 Jan 1997

HAL is a multi-disciplinary open access archive for the deposit and dissemination of scientific research documents, whether they are published or not. The documents may come from teaching and research institutions in France or abroad, or from public or private research centers.
L'archive ouverte pluridisciplinaire HAL, est destinée au dépôt et à la diffusion de documents scientifiques de niveau recherche, publiés ou non, émanant des établissements d'enseignement et de recherche français ou étrangers, des laboratoires publics ou privés. 


\title{
Short Communication
}

\section{Characterizing the Nature of the Smectic A-Smectic $C$ and Smectic A-Smectic C* Transitions}

\author{
L. Benguigui $\left({ }^{1}\right)$ and P. Martinoty $\left({ }^{2, *}\right)$ \\ ( ${ }^{1}$ Solid State Institute and Department of Physics, Technion-Israel Institute of Technology, \\ 32000 Haifa, Israel \\ $\left({ }^{2}\right)$ Laboratoire d'Ultrasons et de Dynamique des Fluides Complexes $\left({ }^{* *}\right)$, \\ Université Louis Pasteur, 4 rue Blaise Pascal, 67070 Strasbourg Cedex, France
}

(Received 01 October 1996, revised 22 November 1996, accepted 16 December 1996)

PACS.61.30.-v - Liquid crystals

PACS.64.70.Md - Transitions in liquid crystals

\begin{abstract}
We provide a theoretical explanation of the critical (2.e. non-mean-field) behavior of specific heat at the $\mathrm{SmA}-\mathrm{SmC}$ transition, as it has recently been observed on certain compounds. This explanation rests on the Andereck and Swift free energy introducing terms which couple the order-parameter with both layer-compression and density. We show that the behavior of specific-heat depends on the intensity of these couplings, and that the behaviors which can be observed are therefore of the mean-field, Gaussian or 3DXY type.
\end{abstract}

Résumé. - Nous donnons une explication du comportement critique (c-à-d. non-champ moyen) de la chaleur spécifique, qui a été observé récemment sur certains composés. Cette explication repose sur l'énergie libre de Andereck et Swift qui introduit des termes couplant le paramètre d'ordre avec la compression de couche et la densité. Nous montrons que le comportement de la chaleur spécifique dépend de l'intensité de ces couplages et que les comportements qui peuvent être observés sont par suite de type champ moyen, gaussien ou 3DXY.

Two recent papers [1,2] give important information, concerning the nature of the smectic Csmectic A transition: through measurements of the specific heat, these results on two different compounds indicate a critical (i.e. a non-mean-field) behavior. From the symmetry of the order parameter, it was predicted by de Gennes [3] that this transition must belong to the universality class of the superfluid helium transition, the 3DXY model. However, a large amount of experimental data [4] have been well-described by the Landau theory with a sixthorder term, implying that this transition is near a tricritical point. In references [1] and [2], it was claimed that it was very difficult to explain these different behaviors for the same transition: meanfield or critical transition. In this report we present a very simple analysis which permits to explain these different behaviors of the specific heat. This analysis is based on the calculations

(") Author for correspondence

$\left({ }^{* *}\right)$ U.R.A. au C.N.R.S. $n^{\circ} 851$ 
which were developed in reference [5] to show that the ultrasound measurements are more sensitive to fluctuation effects than the specific heat measurements.

One possibility for the appearance of a tricritical point is the coupling of the order parameter with the strains. It is well-known that the quadratic coupling of the strains with the order parameter can bring a second order transition to a first order one [6]. Depending on the strength of the coupling, various possibilities may occur: second order transition, tricritical point or first order transition.

In the case of the SmA-SmC transition, Andereck and Swift [7] introduced terms in the free energy coupling quadratically the smectic $\mathrm{C}$ order parameter to the density and to the strain $x_{3}=\partial u / \partial z$ ( $u$ is the deformation and the direction $z$ is perpendicular to the layers). Their free energy used to study the behavior of sound attenuation and dispersion, can be written in the following form (with the notation of Ref. [5]):

$$
\begin{aligned}
F= & \int \mathrm{d}^{3} r\left[\frac{1}{2} a|\eta|^{2}+\frac{1}{4} b|\eta|^{4}+\frac{1}{2} C_{11}\left(x_{1}+x_{2}\right)^{2}+\frac{1}{2} C_{33} x_{3}^{2}+C_{13}\left(x_{1}+x_{2}\right) x_{3}\right. \\
& \left.+\frac{1}{2} \gamma_{\mathrm{u}} x_{3}|\eta|^{2}-\frac{1}{2} \gamma_{\rho}\left(x_{1}+x_{2}+x_{3}\right)|\eta|^{2}+\frac{1}{2} m_{\perp}\left(\nabla_{\perp} \eta\right)^{2}+\frac{1}{2} m_{z}\left(\nabla_{z} \eta\right)^{2}\right]
\end{aligned}
$$

where $\eta$ is the smectic $\mathrm{C}$ order parameter. $x_{1}$ and $x_{2}$ are defined by $x_{1}=\partial u / \partial x$ and $x_{2}=$ $\partial u / \partial y . m_{\perp}$ (the symbol $\perp$ refers to the plane of the layers), $m_{z}$ and $b$ are positive constants and $a=a_{0}\left(T-T_{\mathrm{c}}\right) . C_{11}, C_{33}$ and $C_{13}$ are the elastic constants of the SmA-phase. $\gamma_{\rho}$ and $\gamma_{\mathrm{u}}$ are parameters which couple the smectic $\mathrm{C}$ order parameter to the density variation and to the layer-spacing gradient. However this free energy is not suitable for analysis of the thermal properties (like the specific heat) since in that case it is the Gibbs free energy $G=F-\Sigma X_{\imath} \cdot x_{\imath}$ (the $X_{2}$ 's are the stresses) which is relevant. As shown in reference [5], $G$ is given by

$$
G=\int \mathrm{d}^{3} r\left[\frac{1}{2} a|\eta|^{2}+\frac{1}{4} b^{*}|\eta|^{4}\right]
$$

where

$$
b^{*}=b[1+\chi]
$$

and

$$
\chi=\frac{2 C_{13} \gamma_{\rho}\left(\gamma_{\rho}-\gamma_{\mathrm{u}}\right)-C_{11}\left(\gamma_{\rho}-\gamma_{\mathrm{u}}\right)^{2}-C_{33} \gamma_{\rho}^{2}}{2\left[C_{11} C_{33}-C_{13}^{2}\right]}
$$

The key result for the following is that $\chi$, and consequently $b^{*}$, is function of the parameters $\gamma_{u}$ and $\gamma_{\rho}$. These two parameters are related to the jump $\Delta$ of the elastic constants at $T_{c}$ by the equations

$$
\begin{aligned}
\Delta C_{11} & =-\frac{\gamma_{\rho}^{2}}{2 b} \\
\Delta C_{13} & =-\frac{\gamma_{\rho}\left(\gamma_{\rho}-\gamma_{\mathrm{u}}\right)}{2 b} \\
\Delta C_{33} & =-\frac{\left(\gamma_{\rho}-\gamma_{\mathrm{u}}\right)^{2}}{2 b}
\end{aligned}
$$

where

$$
\Delta C_{\imath j}=C_{\imath j}\left(T_{c}^{-}\right)-C_{\imath j}\left(T_{c}^{+}\right)
$$

These relations allow $\chi$ to be rewritten as a function either of the $\Delta C_{\imath}$ or of the de Gennes elastic constants $A=C_{11}, B=C_{11}+C_{33}-2 C_{13}$ and $C=C_{11}-C_{13}$.

$$
\chi=\frac{\Delta\left(C_{11} C_{33}-C_{13}^{2}\right)}{C_{11} C_{33}-C_{13}^{2}}
$$




$$
\chi=\frac{\Delta\left(A B-C^{2}\right)}{A B-C^{2}}
$$

The $\chi$ value can be determined experimentally since the elastic constants can be deduced from the velocity measurements through the relationship [8]

$$
\rho V^{2}(\theta)=A-2 B \cos ^{2} \theta+C \cos ^{4} \theta
$$

where $\theta$ is the angle between the normal to the smectic layers and the sound propagation direction.

Inspection of formulas (4) and (6b) shows that $\chi$ is always negative and given with a good approximation (because $A \gg B, C[9]$ ) by $\Delta B / B$. Since $B$ is always positive, $|\chi|$ is smaller than one. As a consequence $b^{*}$ cannot be negative but the possibility that $b^{*}$ may be small remains. Since $\chi$ is a function of the coupling parameters $\gamma_{\mathrm{u}}$ and $\gamma_{\rho}, b^{*}$ can vary from a value near zero (and the transition will be almost a tricritical point) to a finite value. It means that the quadratic coupling cannot transform the order of the transition, contrarily to other examples of phase transitions (in ferroelectricity or magnetism) [6]. This unexpected result is due to the layer structure of the smectic A phase, responsible for the very particular properties of this phase.

To determine the nature of a second order transition, it is usual to calculate the width of the critical region with the help of the Ginsburg criterion. This width is given by [5]:

$$
\Delta T_{\mathrm{c}}=\frac{k^{2} T_{\mathrm{c}}}{(16 \pi)^{2}\left(\Delta C_{\mathrm{p}}\right)^{2} \xi_{0 \perp}^{4} \xi_{0 \|}^{2}}
$$

where $\xi_{0 \perp}$ and $\xi_{0\|\|}$ are the bare correlation lengths for order fluctuations perpendicular and parallel to the director respectively, and $\Delta C_{\mathrm{p}}$ the jump of the specific heat. This jump, deduced from equation (2), is given by:

$$
\Delta C_{\mathrm{p}}=C_{\mathrm{p}}\left(T_{\mathrm{c}}^{-}\right)-C_{\mathrm{p}}\left(T_{\mathrm{c}}^{+}\right)=\frac{T_{\mathrm{c}} a_{0}^{2}}{2 b^{*}}
$$

Since $\Delta C_{\mathrm{p}} \sim 1 / b^{*}$, the width of the critical region is proportional to $\left(b^{*}\right)^{2}$. Thus a small change in $b^{*}$, may bring an important variation of $\Delta T_{c}$ and this explains the diversity of behaviors reported for the $\mathrm{SmA}-\mathrm{SmC}$ transition. If $b^{*}$ is very small, $\Delta T_{\mathrm{c}}$ will be also small such that the specific heat behavior will be in accordance with the mean field theory of the tricritical point. However, if $b^{*}$ is large enough, $\Delta T_{\mathrm{c}}$ will get a significant value and deviations from the mean field theory will be detected giving Gaussian (as in [2]) or 3DXY (as in [1]) behaviors.

The critical or non critical nature of the transition is detected in a decisive manner by measuring the elastic constants through ultrasonic measurements. The relevant free energy for the $C_{\imath \jmath}$ 's is (1) and the width of the critical region is now proportional to $b^{2}$ which is always larger than $\left(b^{*}\right)^{2}[5]$. It is why the compounds TBBA and $\overline{8} \mathrm{~S} 5$ have a mean-field-like behavior from the specific heat measurements $[10,11]$, but distinct critical behavior from the ultrasonic measurements $[12,13]$.

We mentioned above that the mechanism of Andereck and Swift cannot bring the transition to be first order. However, first order SmA-SmC transitions have been observed, as mentioned in reference [2], but this results from another type of coupling. One example of such coupling is given in [14].

In conclusion, we showed that the different behaviors (Landau, Gaussian, 3DXY) which were observed in the specific heat are easily explained by means of the free energy of Andereck and Swift. The specific heat behavior is simply related to the strength of the coefficients which 
couple the $\mathrm{SmC}$ order parameter with the layer-compression and with the density. We emphasize that there is no reason why the $\mathrm{SmA}-\mathrm{SmC}$ transition should be close to a tricritical point, generically. It is only because the presence of the particular coupling mentioned above that such a tricritical transition could appear. Therefore, the several examples of tricritical behavior which were reported do not represent an intrinsic property of the $\mathrm{SmA}-\mathrm{SmC}$ transition.

\section{References}

[1] Ema K., Watanabe J., Takagi A. and Hao H., Phys. Rev. E 52 (1995) 1216.

[2] Reed L., Stoebe T. and Huang C.C., Phys. Rev. E 52 (1995) R2157.

[3] de Gennes P.G., Mol. Crys. Liq. Cryst. 21 (1973) 49.

[4] See references in [1] and [2]. For exceptions, see references [6] and [7] in [1].

[5] Benguigui L. and Martinoty P., Phys. Rev. Lett. 63 (1989) 774.

[6] Devonshire L.F., Adv. Phys. 3 (1954) 85.

[7] Andereck B.S. and Swift J., Phys. Rev. A 25 (1982) 1084.

[8] See for example Martin P.C, Parodi O. and Pershan P.S., Phys. Rev. A 6 (1972) 2401.

[9] Collin D., Gallani J.L. and Martinoty P., Phys. Rev. A 34 (1986) 2255.

[10] Das P., Ema K. and Garland C.W., Liq. Cryst. 4 (1989) 205.

[11] Schantz C.A. and Johnson D.L., Phys. Rev. A 17 (1978) 1504.

[12] Collin D., Gallani J.L. and Martinoty P., Phys. Rev. Lett. 61 (1988) 102.

[13] Collin D., Moyses S., Neubert M.E. and Martinoty P., Phys. Rev. Lett. 73 (1994) 983.

[14] Benguigui L., J. Phys. Colloq. France 40 (1979) C3-419. 